\title{
APRENDIZAGEM COM MOBILIDADE (M-LEARNING) NOS PROCESSOS DE ENSINO E DE APRENDIZAGEM: REFLEXÕES E POSSIBILIDADES*
}

\author{
Ms. Paulo Gaspar Graziola Junior - UNISINOS - pgraziola@ gmail.com ${ }^{* * *}$
}

RESUMO - Este artigo relata resultados de uma pesquisa na qual propus investigar "como as Tecnologias Móveis Sem Fio (TMSF) podem contribuir para práticas pedagógicas numa perspectiva dialógica, colaborativa e cooperativa”. Como recorte, esse artigo contempla as implicações do uso das TMSF nos processos de ensino e aprendizagem, uma das unidades de análise. A metodologia utilizada foi estudo de caso - entendendo as práticas pedagógicas com o uso das TMSF no contexto da aprendizagem com mobilidade como o caso a ser pesquisado. Assim, a partir da análise de duas situações de práticas pedagógicas, a primeira com o Grupo de Sistemas de Informação (GSI) da Universidade do Vale do Rio dos Sinos, por meio de observação direta e, a segunda com um grupo de Secretariado da área de Ciências Humanas, da mesma universidade. Os resultados são válidos para provocar a reflexão de professores/orientadores sobre a necessidade de desenvolver práticas pedagógicas capazes de dar conta das especificidades relacionadas ao processo de ensino e de aprendizagem nessa modalidade, superando o paradigma educacional vigente $e$ estando mais perto do contexto dos sujeitos.

Palavras-chave: aprendizagem com mobilidade. tecnologias móveis sem fio. práticas pedagógicas. ensino. aprendizagem.

ABSTRACT - This article tells resulted of a research in which I considered to investigate "how the wireless mobile technologies (WMTs) can contribute to the teaching practices in a dialogical, collaborative and cooperative perspective". The methodology that was used to the development of the research was the study case understanding the teaching practices with the use of the WMTs in the mobile-learning context. So, from the analysis of two teaching practice situations with WMT, the first with the Information Systems Group of the University of the Rio dos Sinos Valley, using direct observation and the second one with a group of secretariat of Human Sciences Department, of the same university. The results are valid to provoke the reflection of the teacher/ the orientation role on the necessity to develop practical pedagogical capable to give to account of the specificities related to the process of education and learning in this modality, surpassing the effective educational paradigm and being more close to the context them citizens.

Key words: mobile learning. wireless mobile technologies. teaching practices. teaching. learning.

\footnotetext{
* Este trabalho faz parte da dissertação de mestrado defendida pelo Programa de Pós-Graduação em Educação da Unisinos (GRAZIOLA JUNIOR, 2009). Contou com o apoio financeiro (bolsista) do Conselho Nacional de Pesquisa (CNPq).

** Mestre em Educação pela Universidade do Vale do Rio dos Sinos - UNISINOS; Graduação em Ciência da Computação - UNISC; Participante do Grupo de Pesquisa Educação Digital - GPe-du UNISINOS.
} 


\section{1 - Introdução}

As mudanças no contexto social e educacional, provocadas pelo constante avanço tecnológico e científico, e as exigências do mercado de trabalho por pessoas mais qualificadas e capazes de aprender e resolver problemas cooperativamente são crescentes. Além disso, o crescimento exponencial das informações e a grande proliferação destas têm resultado na rápida defasagem dos conhecimentos, o que exige uma constante atualização. Assim, conforme Silverman (1995), a aprendizagem passa a ser uma atividade para a vida toda, e o sujeito precisa desenvolver habilidades de "aprender a aprender" e "aprender cooperativamente", para que o processo de aprendizagem seja efetivo.

Poucas Instituições de Ensino têm conseguido vivenciar práticas inovadoras. Essas práticas podem ampliar os espaços para além da sala de aula formal, por minimizar as barreiras impostas pelo tempo e pelo espaço, ajudando na ampliação dos processos de ensino e aprendizagem e na criação de comunidades de aprendizagem. Mas como criar condições para que isto possa ocorrer? Que Tecnologias Digitais (TDs) podem ajudar a melhor aproveitar o tempo que temos? Como propiciar práticas pedagógicas para além do contexto formal físico e presencial? As Tecnologias da Informação Móveis e Sem Fio (TMSF), aliadas a metodologias adequadas à natureza desse meio, podem representar uma possibilidade de resposta a essas indagações?

As potencialidades da TMSF precisam ser compreendidas e avaliadas para ampliar e melhorar as práticas pedagógicas e, consequientemente, os processos de ensino e de aprendizagem.

Por meio da análise das interações dos sujeitos que participaram do estudo, procuramos compreender como eles se relacionam e quais as especificidades dos processos elaborados. Também, as formas pelas quais os sujeitos atingiram os resultados encontrados, e a maneira pela qual se manifestaram os diferentes tipos de interações entre os interlocutores do processo de aprendizagem via m-learning.

Novos meios tecnológicos, nesse caso, as TMSF, por si só não se constituem em inovações nos processos de ensino e de aprendizagem, na medida em que a inovação implica na superação de paradigmas. Essa compreensão tem povoado a mente de professores/pesquisadores que se apropriam das tecnologias, utilizando-as de forma crítica, refletindo sobre o que representam no contexto do desenvolvimento humano, das organizações e das sociedades, deslocando a compreensão equivocada de que a tecnologia é a inovação, para compreendê-la como propulsora do surgimento de inovações, as quais surgem no conhecer, portanto no viver e conviver.

Nesse artigo, toma-se como objeto a prática pedagógica com o uso de TMSF no contexto da aprendizagem com mobilidade e como recorte, as implicações nos processos de ensino e aprendizagem, uma das unidades de análise da pesquisa.

Primeiramente, é realizada uma contextualização da aprendizagem com mobilidade (m-learning), logo após, é descrita a metodologia empregada; as características dos casos estudados, seguidas da análise dos dados e achados e, por fim, as considerações finais e trabalhos futuros.

\section{2 - Aprendizagem com mobilidade (m-learning)}

A crescente necessidade de mobilidade de pessoas, de acesso e troca de informações em qualquer tempo e espaço, de compartilhamento de idéias, de experiências e de conhecimento, além da necessidade de ampliar os espaços formais de 
educação, oportunizam o emprego da emergente aprendizagem com mobilidade $(\mathrm{m}$ learning).

A aprendizagem com mobilidade (m-learning) é possibilitada por meio do desenvolvimento e aplicação de TMSF, que são dispositivos computacionais portáteis, tais como PDAs (Assistentes Pessoais Digitais), palmtops, laptops, smartphones, dentre outros, que utilizam redes sem fio. $\mathrm{O}$ uso de TMSF, aliado à interação com atores humanos distantes geograficamente e fisicamente, pode propiciar que ocorram processos de ensino e de aprendizagem.

Sob o ponto de vista tecnológico, conforme Meirelles et al. (2004, p. 4):

[...] duas categorias de sistemas finais começam a ganhar popularidade mundial. A mais antiga é representada pelos $P D A$ s, com uma estimativa no Brasil de aproximadamente dois milhões de usuários. A segunda categoria é representada pelos chamados telefones inteligentes (smartphones), que além das funcionalidades dos bons $P D A$ s, permitem a comunicação por voz, navegação na internet, disponibilizando em alguns modelos conexões Bluetooth e Wi-Fi.

Meirelles et al. (2004, p.5) complementa, uma vez que os recursos destinados à infra-estrutura de transmissão, existentes nas redes de comunicação móvel tornam-se disponíveis, novos desafios e necessidades de estudo surgem considerando os recursos computacionais dos dispositivos portáteis como, por exemplo, dos PDAs. Aplicativos consagrados em ambientes de informática onde predominam os chamados computadores de mesa, oferecem aos usuários a possibilidade de conversão e manuseio de informações em formato multimídia.

Sistemas gerenciadores de aprendizagem com código aberto poderão ter suas funcionalidades ampliadas, suportando novas aplicações destinadas a migrar ferramentas de comunicação para PDAs (Correio Eletrônico, Fórum, Diário de Bordo), acrescidas de rotinas para sincronização de dados com o servidor. Desta forma, tanto o trabalho off-line como o trabalho on-line poderá ser efetuado sem restrição de tempo e de espaço geográfico.

Para Reinhard et al. (2007, p. 1),

aprender com mobilidade não é uma idéia nova - a possibilidade de aprender em qualquer lugar e a qualquer momento sempre foi buscada e potencializada com ferramentas como livros, cadernos e outros instrumentos móveis (portáteis) que existem há muito tempo. O que hoje ocorre é que as TMSF podem contribuir para a Aprendizagem com Mobilidade por disponibilizarem aos sujeitos o acesso rápido a uma grande e diversificada quantidade de informações, viabilizando seu recebimento e envio (quando associadas à Internet); além disso, essas tecnologias promovem a comunicação e a interação entre pessoas distantes geograficamente e temporalmente, de uma maneira sem precedentes.

Marçal et al. (2005, p. 3) colocam que,

[...] o m-learning surge como uma importante alternativa de ensino e treinamento à distância, na qual podem ser destacados os seguintes objetivos: - melhorar os recursos para o aprendizado do aluno, que poderá contar com um dispositivo computacional para execução de tarefas, anotação de idéias, consulta de informações via internet, registro de fatos através de câmera digital, gravação de sons e outras funcionalidades existentes;

- prover acesso aos conteúdos didáticos em qualquer lugar e a qualquer momento, de acordo com a conectividade do dispositivo;

- aumentar as possibilidades de acesso ao conteúdo, incrementando e incentivando a utilização dos serviços providos pela instituição, educacional ou empresarial; 
- expandir o corpo de professores e as estratégias de aprendizado disponíveis, através de novas tecnologias que dão suporte tanto à aprendizagem formal como à informal;

- expandir os limites internos e externos da sala de aula ou da empresa, de forma ubíqua;

- fornecer meios para o desenvolvimento de métodos inovadores de ensino e de treinamento, utilizando os novos recursos de computação e de mobilidade.

Já Saccol e Reinhard (2005) comentam que o uso das TMSF pode trazer uma série de vantagens, mas cada vantagem analisada precisa considerar possíveis contrapontos, por exemplo: é possível aos usuários da tecnologia despender mais tempo em campo e menos tempo em tarefas de "bastidores" no escritório ou trabalhar em determinados "tempos mortos", muito embora as limitações técnicas e ergonômicas das TMSF e o próprio contexto do trabalho móvel possam por vezes impor restrições a isso; é possível localizar pessoas e ser localizado com maior rapidez e freqüência, assim como trocar dados de forma mais rápida e fácil, em diferentes locais e momentos, o que contribui para os processos de tomada de decisão - por outro lado uma sobrecarga, muitas vezes gerada pela fácil replicação de dados, aumenta consideravelmente.

A seguir é realizada uma descrição da metodologia empregada.

\section{3 - Metodologia}

A natureza da pesquisa fundamentou-se numa abordagem exploratória e qualitativa. Optou-se por utilizar o método de "Estudo de Caso" por melhor se adequar ao contexto da pesquisa. O estudo de caso é uma estratégia de pesquisa que contribui para a pesquisa do objeto em relação a seu contexto. Assim, o estudo de caso serviu como recorte metodológico e a pesquisa participante serviu como modo de interlocução entre os sujeitos, especificamente no segundo caso. Streck e Brandão (2006, p. 52) colocam que "na pesquisa participante parto de um duplo reconhecimento de confiança em meu 'outro', naquele que procuro transformar de 'objeto de minha pesquisa' em 'cosujeito' de nossa investigação".

O primeiro estudo de caso se refere ao processo formativo constituído por um Workshop chamado "Tecnologias Móveis e Sem Fio: o novo paradigma da ubiqüidade e suas possibilidades de aplicação", desenvolvido junto a uma equipe de colaboradores da GSI (Gerência de Sistemas de Informação) da UNISINOS. Ao todo participaram treze colaboradores da GSI.

O segundo estudo de caso correspondeu a uma oficina temática desenvolvida com uma equipe do SCH (Secretariado de Ciências Humanas) da UNISINOS. Este segundo Estudo de Caso teve caráter de pesquisa participante, conforme citado anteriormente e se refere ao desenvolvimento e análise de uma prática pedagógica com o uso das TMSF numa perspectiva dialogada, colaborativa e cooperativa (GRAZIOLA JUNIOR, 2009). Ao todo participaram doze colaboradores da SCH.

A pertinência da escolha dos casos está no fato das práticas pedagógicas se desenvolverem com distintos grupos de sujeitos que atuam de forma não fixa: os chamados trabalhadores móveis, cuja interação é tensionada pela introdução de um novo elemento, no caso ligado às TMSF.

Para a pesquisa empírica, foi utilizado um protótipo, o Ambiente Virtual de Aprendizagem com Mobilidade (AVAM) denominado COMTEXT, modelado e construído no contexto da pesquisa interdisciplinar "Aprendizagem com Mobilidade no 
contexto organizacional" ". Em ambas capacitações foram utilizados os seguintes materiais/instrumentos: observação, diário, e-mail, chat, fórum, skype, questionários.
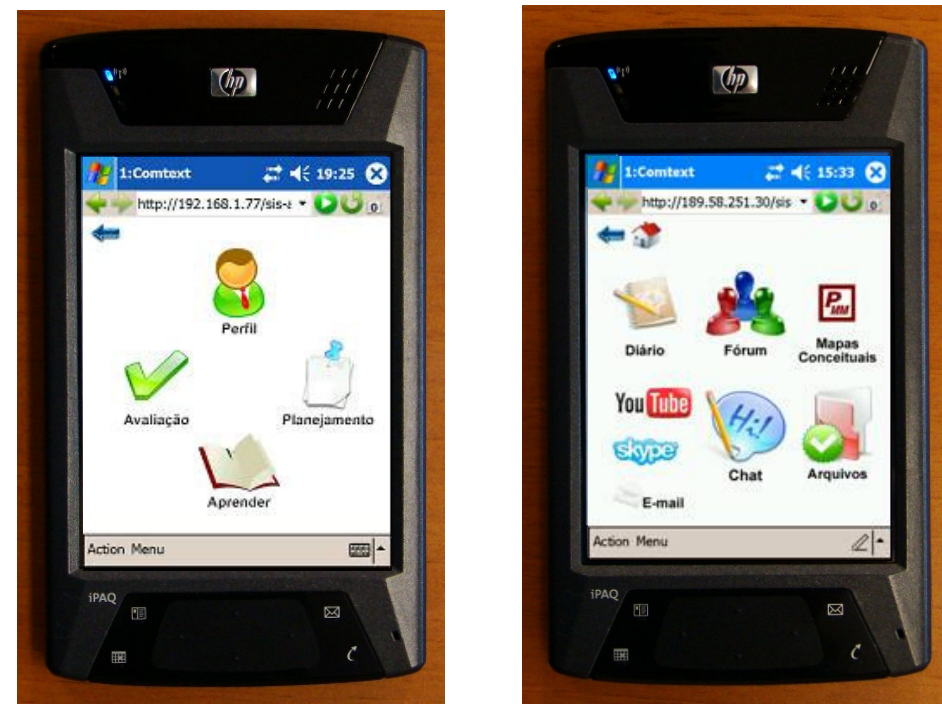

Figura 1 - Protótipo COMTEXT (Ambiente de Competência em Contexto) - Módulo Principal e Módulo Aprender, respectivamente.

Do ponto de vista da equipe do desenvolvimento do projeto, para que o $\mathrm{m}$ learning possa oferecer novas possibilidades de aprendizagem num contexto de mobilidade, é necessário, fundamentalmente, repensar os paradigmas educacionais, as concepções de aprendizagem, bem como as práticas pedagógicas desenvolvidas. Atualmente, um número significativo de sujeitos-trabalhadores passa por inúmeras experiências educacionais e verificam que muitas vezes elas são incipientes e não atendem as suas necessidades de aprendizagem. Acredita-se que um modelo que esteja fundamentado por uma concepção epistemológica interacionista-construtivistasistêmica possa dar conta de ajudar os sujeitos-trabalhadores a desenvolver competências necessárias para atuar num contexto de mobilidade (GRAZIOLA JUNIOR, 2009).

Assim, o desenvolvimento do COMTEXT está fundamentado na concepção epistemológica interacionista-construtivista-sistêmica, na qual o sujeito num processo de interação com o meio físico e social, constrói conhecimentos, entendendo que o processo de conhecimento é compreendido como sendo um todo integrado cujas propriedades fundamentais têm sua origem nas relações entre suas partes. Constituído de sub-sistemas que se inter-relacionam formando uma rede em que estes estão interligados e são interdependentes, de forma que o todo é maior que a soma das partes, pois envolve as partes e fundamentalmente as relações entre elas.

A seguir são descritas as características dos dois casos que fizeram parte da presente pesquisa.

\footnotetext{
${ }^{1}$ Integram a equipe: Prof. Dr. Nicolau Reinhard - FEA/USP (reinhard@usp.br) - Coordenador, Profa. Dra. Amarolinda Zanela Saccol - UNISINOS (aczanela@unisinos.br), Profa. Dra. Eliane Schlemmer UNISINOS (elianes@unisinos.br), e Prof. Dr. Jorge Luis V. Barbosa - UNISINOS (jbarbosa@unisinos.br) e Prof. Dr. Steinar Krisoffersen - Østfold University College - Noruega -

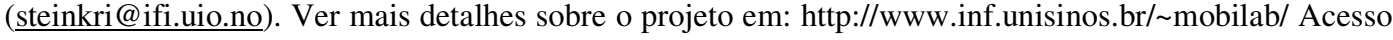
em: 20/03/2009.
} 


\section{1 - Características do Workshop e da Oficina Temática}

Ambas as capacitações foram desenvolvidas no contexto da Aprendizagem com mobilidade, por meio do uso de iPAQs HP 4700 Pocket PC e do ambiente COMTEXT.

O Workshop "Tecnologias Móveis e Sem Fio: o novo paradigma da ubiqüidade e suas possibilidades de aplicação" teve como objetivo promover a compreensão e discussão do novo paradigma da ubiqüidade e suas possíveis aplicações para a UNISINOS. A escolha se deu, em função de serem trabalhadores móveis, isto é, exercerem suas atividades profissionais em diferentes pontos físicos da UNISINOS e por utilizarem diariamente a tecnologia, uma vez que atuam gerenciando todo o Sistema de Informação da Universidade. Dessa forma, além de adquirirem conhecimentos sobre a temática do Workshop, puderam contribuir no teste do protótipo COMTEXT, auxiliando assim em melhorias e ajustes para práticas posteriores.

O Workshop teve a duração de duas semanas, num total de 15 horas, sendo dois encontros presenciais físicos de 1 hora e 30 minutos de duração (um encontro de abertura e outro de encerramento) e os demais, totalizando 12 horas, realizado de forma presencial digital virtual (online). Nos encontros presencias digitais virtuais, que ocorreram num total de oito dias, as atividades foram guiadas por perguntas-eixo (tópicos de estudo/discussão), um tópico trabalhado por dia.

Como atividade prática final, o qual faria parte da avaliação do Workshop, foi proposta a elaboração de um projeto (hipotético) em grupos considerando possibilidades de aplicação dessas tecnologias móveis e sem fio para as atividades da UNISINOS.

A partir do primeiro caso (Workshop), algumas questões serviram de elementos para subsidiar o desenvolvimento da Oficina Temática.

A Oficina temática intitulada "Diálogo, Colaboração e Cooperação no contexto do trabalho coletivo" teve como objetivo promover a compreensão dos conceitos de Diálogo, Colaboração e Cooperação e sua importância para o desenvolvimento de atividades que exigem articulação em equipe, por meio de vivências de práticas pedagógicas colaborativas no contexto da Aprendizagem com Mobilidade.

As atividades foram desenvolvidas na modalidade b-learning, num total de 30 horas, quando tínhamos alguns encontros presenciais físicos (1h30min de duração dois por semana) e os demais presenciais digitais virtuais (o restante das horas trabalhadas), utilizando o Ambiente COMTEXT.

Durante a prática pedagógica, foi proposta a elaboração de um projeto de aprendizagem (FAGUNDES et al., 1999) cujo tema emergisse de um consenso conforme necessidades individuais e de grupo e, que fizesse parte de seus contextos profissionais, culminando numa posterior aplicação prática no dia-a-dia. Para desenvolver o projeto de aprendizagem deveriam encontrar parceiros (entre os colegas), conforme interesses e curiosidades compartilhados sobre o problema a ser resolvido. Assim, os participantes fizeram primeiramente um levantamento de suas dúvidas temporárias e suas certezas provisórias em relação ao problema que gerou o projeto.

\section{4 - Análise de dados e achados}

O uso da mobilidade nos processos de ensino e de aprendizagem implica, necessariamente, numa mediação pedagógica eficiente, na qual o professor tem a função de orientador, problematizador. A ele cabe provocar a discussão dos sujeitos, a participação, cabe acompanhar e analisar a construção do conhecimento por meio da 
interação realizada nos espaços disponibilizados no ambiente, no caso, o ambiente COMTEXT.

Foi possível observar uma similaridade em relação à forma como foi desenvolvida a mediação pedagógica dos professores-orientadores, pois em ambos os casos, com os sujeitos da GSI, onde se utilizou em praticamente todos os encontros a ferramenta chat, e com os sujeitos do $\mathrm{SCH}$, onde se utilizou nos encontros a ferramenta fórum, o que se evidenciou foi uma mediação pedagógica quanto a três aspectos distintos (em relação ao conteúdo, em relação à participação dos sujeitos e em relação à organização do processo), porém interligados, se aproximam do que (RIOS, 2002) coloca, ao referir que o objetivo do professor na atualidade é justamente superar a fragmentação do conhecimento, da comunicação e das relações por meio de um diálogo ou de uma parceria. Assim, dentro dessa perspectiva, a atuação do professor-orientador não deve se limitar a fornecer informações aos alunos, mas assumir a mediação das interações entre aluno-informação-dispositivo-aluno, de modo que esse diálogo entre professor e alunos, possibilite a construção do conhecimento em um ambiente desafiador.

Nesse processo de mediação pedagógica, foi evidenciada ainda, pelos sujeitos do $\mathrm{SCH}$, a disponibilidade e a dinâmica utilizada pelo professor-orientador, o estabelecimento de uma relação entre a didática do professor-orientador e o seu comprometimento perante a Oficina Temática e a importância da mediação pedagógica, no sentido de criar um clima agradável e saudável que propiciasse a aprendizagem. As evidências se aproximam de (FREIRE, 1987) quando coloca que o educador já não é o que apenas educa, mas o que enquanto educa, é educado, em diálogo com o educando que, ao ser educado, também educa. Ambos, assim, se tornam sujeitos do processo em que crescem juntos e em que os "argumentos de autoridade" já nada valem. Freire e Shor (2006) complementam ainda que o diálogo sela o ato de aprender, que nunca é individual, embora tenha uma dimensão individual.

Em relação à reflexão dos sujeitos sobre o seu processo de aprendizagem, no contexto do uso das TMSF, os sujeitos do SCH quando questionados se a forma como a capacitação foi desenvolvida com o uso do COMTEXT, contribuiu para uma aprendizagem mais significativa, vincularam a aprendizagem às práticas e processos de mediação pedagógica adotadas pelo professor-orientador, presumindo assim mais uma vez a relação dialógica professor-aluno, e à reflexão em relação ao trabalho diário. As respostas se aproximam do que diz Estabel et al., 2003), ao referir que para que ocorra a aprendizagem, é necessário um processo de troca com o outro. A aprendizagem cooperativa apoiada por computador deve ser uma estratégia educativa na qual dois ou mais sujeitos constróem o seu conhecimento a partir da discussão, do diálogo, da reflexão e da tomada de decisão. Para que esse processo ocorra, professores e alunos devem estar dispostos a além de colaborar, construir conhecimentos conjuntamente, ter objetivos comuns.

Esses registros enfatizam ainda a importância da relação professor-aluno na prática pedagógica, se aproximando de uma prática pedagógica dialógica, que, conforme Fernandes (2006, p. 376) coloca, refere-se a uma "prática caracterizada pela aprendizagem do professor com seus alunos. Estes, por meio do diálogo, aprendem também que o compromisso da parceria é fundamental em uma relação humana horizontal, que não exclui do professor a responsabilidade de direção do processo de ensinar e aprender, nem exime o aluno da responsabilidade de ultrapassar os limites de sua prática, recriando o espaço-tempo da reflexão e da cultura em relações democráticas e éticas." Assim, quando há um comprometimento de parceria na relação professoraluno, permeado por um constante diálogo, numa relação democrática e ética, no 
momento da prática pedagógica, permite que se criem espaços onde o sujeito possa refletir sobre o seu pensar e sobre seu processo de aprendizagem.

Em relação às percepções dos sujeitos da GSI e do SCH quanto às implicações do uso da mobilidade no processo de ensino e de aprendizagem. Os sujeitos da GSI evidenciaram que implica em: na necessidade de aprender a utilizar o equipamento (hardware e software), em pensar na oferta de serviços para os alunos, sendo que alguns apontam ainda como implicações a motivação para a pesquisa e para a colaboração. Marçal et al. (2007) colocam como um dos objetivos da aprendizagem com mobilidade, aumentar as possibilidades de acesso ao conteúdo, incrementando e incentivando a utilização dos serviços providos pela instituição, educacional ou empresarial. Além disso, o ambiente cooperativo deve ser voltado para a aprendizagem do sujeito e permitir que torne-se um agente ativo que (re)constrói seu conhecimento na interação com o objeto de conhecimento, seja ele do meio físico ou social. Assim, as TMSF (e o ambiente COMTEXT em particular) tornam-se ferramentas que podem oportunizar a interação entre sujeito-tecnologia.

Para os sujeitos do $\mathrm{SCH}$, implica em: muitas aprendizagens novas, na possibilidade efetiva de interação, de diálogo e de trocas; em aprender a organizar e administrar o tempo e implica em comprometimento e reflexão. Fica evidenciado, que as percepções dos sujeitos (GSI e $\mathrm{SCH}$ ) vão ao encontro das propostas de suas capacitações, fazendo relações com suas implicações, de um lado o Workshop que objetivava, a compreensão e discussão de TMSF e suas possíveis aplicações para a UNISINOS, outro lado a Oficina Temática que objetivava promover a compreensão dos conceitos de diálogo, colaboração e cooperação e sua importância para o desenvolvimento de atividades que exigem articulação em equipe, por meio de vivências de práticas pedagógicas colaborativas no contexto da aprendizagem com mobilidade.

\section{5 - Considerações e Trabalhos Futuros}

A partir da análise dos dados e dos achados, seguem algumas reflexões e possibilidades do uso das TMSF nos processos de ensino e aprendizagem, uma das unidades de análise da pesquisa.

Possibilitar a criação de ambientes que possibilitem ao sujeito aprendiz continuar a aprender, mesmo estando fora da instituição/lugar formal de ensino e/ou seja, em contexto de mobilidade.

Haver um planejamento aberto, flexível, isto é, uma dinâmica que se adapte e respeite fundamentalmente o contexto dos sujeitos envolvidos nesse processo, pois ao usaram as TMSF, dentro da sua prática cotidiana, estes ficam limitados ao espaço de trabalho diário (físico) e à questão "tempo".

Possibilitar espaços de reflexão, por meio do saber construído (as aprendizagens) do sujeito, no plano individual, e por meio da interação e do diálogo com os colegas e o professor-orientador, no plano coletivo, além da reflexão desses sujeitos da influencia dessas TMSF nas suas práticas diárias, para desse modo, revisarem e reconfigurarem suas práticas.

Propiciar atividades dando um valor especial às sensações, subjetividades, impressões, desejos e afetos dos sujeitos, imbricados no processo educativo, não esquecendo os objetivos de seu uso, sobretudo no contexto da mobilidade, devido às 
restrições técnicas e ergonômicas dos dispositivos móveis, não se esquecendo de prevalecer as questões didático pedagógicas em relação às questões tecnológicas.

Pensar numa forma de adaptar seu melhor seu uso ao contexto dos sujeitos, para não acabar limitando a participação por parte dos sujeitos e a própria adaptação dessas tecnologias.

Propiciar encontros (sejam presenciais-físicos ou online) como espaços de apoio e de "encontro", principalmente para resolução de dúvidas, trocas e questionamentos.

Pensar na mediação pedagógica, numa perspectiva dialógica entre aluno e professor, na qual o professor tem a função de orientador, problematizador, não limitando o papel do professor a somente fornecer informações, mas assumindo a mediação das interações entre aluno-informação-dispositivo-aluno.

Pensar no uso de projetos de aprendizagem que podem configurar como possibilidade de prática pedagógica que instiga a ação, a interação dos sujeitos, mediada pelo professor-orientador, contribuindo para a formação de sujeitos autônomos, reflexivos, e com alto nível de autoria e ainda propiciar processos de cooperação.

Perceber como alguns desafios tecnológicos e ergonômicos, de tempo e de espaço, podem afetar ou restringir o seu uso nas práticas pedagógicas no contexto da mobilidade.

Ter o fator "tempo" como sinalizador, pois não basta somente prover acesso aos conteúdos em qualquer lugar e a qualquer momento, mas propiciar espaços e um tempo hábil para que os sujeitos possam ler, estudar, agir, interagir, enfim construir conhecimento.

Estar atento ao contexto de cada sujeito, não esquecendo as restrições dos "tempos mortos" (tempos livres), e sua dualidade, pois se por um lado é interessante o uso desses "tempos mortos", por outro, podem trazer uma série de riscos, como por exemplo, uma sobrecarga de informações e um controle informacional.

Não esquecer do fator "espaço físico (contexto)" que pode de certa forma "limitar" a mobilidade, o uso efetivo dela, devido principalmente às restrições tecnológicas de acesso a rede em diversos pontos.

Possibilitar o uso "efetivo" da mobilidade, que além de poder expandir os limites das práticas podem ainda propiciar outras possibilidades, como por exemplo, localização e formação de grupos conforme afinidades dos sujeitos, anotações de observações em saídas a campo, entre outras.

Desta forma, esta investigação consistiu em um espaço de experiência e convite à reflexão sobre o uso das TMSF na educação sem apresentar medidas pontuais e passageiras, soluções imediatas de trabalho que apontam a máquina como centro do processo de aprendizagem. A proposta foi centrada na interação sujeitos-tecnologiasujeitos, que viabiliza o processo de produção de conhecimentos democraticamente nas diferentes áreas do conhecimento. Neste artigo buscou-se observar as potencialidades motivadas pela interação dos sujeitos com essa tecnologia.

Como possibilidades de continuação, apontam-se alguns questionamentos que podem guiar futuras investigações: Quais as possibilidades de trabalhar num contexto móvel sem limitações de acesso à rede e mobilidade, por exemplo, com acesso à rede 3G? Como os sujeitos poderiam realizar saídas de campo com dispositivos móveis? De que forma isso contribuiria para o ensino e a aprendizagem do sujeito? Quais as possibilidades em trabalhar por localização de grupos por interesse, conforme suas definições de perfil gravadas previamente? Quais as possibilidades de uso de outros dispositivos, como por exemplo, o iPhone, que permite outras funcionalidades além das oferecidas pelos PDAs? 
6 - Referências

ESTABEL, L. B.; MORO, E. L. da S.; SANTAROSA, L. M. C.i. Abordagens de Cooperação na Utilização de Ambiente de Aprendizagem Mediado por Computador pelos Portadores de Necessidades Educacionais Especiais com Limitação Visual. Informática na Educação: teoria \& prática, Porto Alegre, 2003.

FAGUNDES, Léa da Cruz; SATO, Luciane Sayuri; MAÇADA, Débora Laurino. Projeto? O que é? Como se faz? In: Aprendizes do Futuro: as inovações começaram! Coleção Informática para a mudança na Educação. Brasília, MEC, 1999. Disponível em: <http://mathematikos.psico.ufrgs.br/textos.html> Acesso em: $10 \mathrm{de}$ Ago. 2008.

FERNANDES, Cleoni Maria Barbosa. In: MOROSINI, Marília Costa; ROSSATO, Ricardo (Ed.) et al. Enciclopédia de pedagogia universitária: glossário. Brasília: INEP, 2006.

FREIRE, Paulo. Pedagogia do oprimido. 17. ed. Rio de Janeiro: Paz e Terra, 1987.

; SHOR, Ira. Medo e ousadia: o cotidiano do professor. 11. ed. São Paulo: Paz e Terra, 2006.

GRAZIOLA JUNIOR, Paulo Gaspar. Aprendizagem com mobilidade na perspectiva dialógica: reflexões e possibilidades para práticas pedagógicas. São Leopoldo (RS): UNISINOS, 2009. Dissertação (Mestrado em Educação), Universidade do Vale do Rio dos Sinos, 2009.

MARÇAL, Edgar; SANTOS, Raniery; VIDAL, Creto, ANDRADE, Rossana; RIOS, Riverson. MuseuM: Uma Aplicação de m-learning com Realidade Virtual. Seminário Integrado de Software e Hardware. São Leopoldo: Unisinos, 2005a. Disponível em: http://www.lia.ufc.br/ great/artigos/museuM.pdf. Acesso em: 10 de Jun. 2007.

MEIRELLES, Luiz Fernando Tavares; TAROUCO, Liane Margarida Rockenbach; ALVES, Carlos Vinicius Rasch. Telemática Aplicada à Aprendizagem com Mobilidade. In: RENOTE - Revista Novas Tecnologias na Educação, Porto Alegre. V. $2 \mathrm{~N}^{\mathrm{o}}$ 2, novembro, 2004.

REINHARD, Nicolau; SACCOL, Amarolinda Zanela; SCHLEMMER, Eliane; BARBOSA, Jorge; KRISTOFFERSEN, Steinar. Aprendizagem com mobilidade no contexto organizacional. Disponível em: http://www.inf.unisinos.br/ mobilab/ Acesso em: 20 de Abr. 2007.

RIOS, Terezinha Azerêdo. Compreender e ensinar: por uma docência da melhor qualidade. 3. ed. São Paulo: Cortez, 2002.

SACCOL, Amarolinda; REINHARD, Nicolau. Processo de Adoção e Decorrências da Utilização de Tecnologias de Informação Móveis e Sem Fio no Contexto Organizacional. In: XXIX EnANPAD, 2005, Brasília. Anais do XXIX EnANPAD 2005, v. 1, 2005, p. 1-16.

SILVERMAN, Barry G. Computer Supported Collaborative Learning (CSCL). Computers Education, 1995.

STRECK, Danilo R. e BRANDÃO, Carlos Rodrigues. A pesquisa participante e a partilha do saber: uma introdução. In: STRECK, Danilo R. e BRANDÃO, Carlos Rodrigues (orgs.). Pesquisa Participante: a partilha do saber. Aparecida, SP: Idéias e Letras, 2006. 